\title{
INFINITE FAMILIES OF ISOMORPHIC NONCONJUGATE FINITELY GENERATED SUBGROUPS
}

\author{
F. E. A. JOHNSON
}

\begin{abstract}
Let $\langle\rangle:, L \times L \rightarrow \mathbb{Z}$ be a nondegenerate symmetric bilinear form on a finitely generated free abelian group $L$ which splits as an orthogonal direct sum $(L,\langle\rangle,) \cong\left(L_{1},\langle\rangle,\right) \perp\left(L_{2},\langle\rangle,\right) \perp\left(L_{3},\langle\rangle,\right)$ in which $\left(L_{1},\langle\rangle,\right)$ has signature $(2,1),\left(L_{2},\langle\rangle,\right)$ has signature $(n, 1)$ with $n \geq 2$, and $\left(L_{3},\langle\rangle,\right)$ is either zero or indefinite with $\operatorname{rk}_{\mathbf{z}}\left(L_{3}\right) \geq 3$. We show that the integral automorphism group $\operatorname{Aut}_{\mathbf{z}}(L,\langle\rangle$,$) contains an infinite family of$ mutually isomorphic finitely generated subgroups $\left(\Gamma_{\sigma}\right)_{\sigma \in \Sigma}$, no two of which are conjugate. In the simplest case, when $L_{3}=0$, the groups $\Gamma_{\sigma}$ are all normal subdirect products in a product of free groups or surface groups. The result can be seen as a failure of the rigidity property for subgroups of infinite covolume within the corresponding Lie group $\operatorname{Aut}_{\mathbf{z}}(L \otimes \mathbf{z} \mathbb{R},\langle,\rangle \otimes 1)$.
\end{abstract}

\section{INTRODUCTION}

The following question arose from the joint work of Ebeling and Okonek on diffeomorphisms of algebraic surfaces.

Question. Let $\langle\rangle:, L \times L \rightarrow \mathbb{Z}$ be a nondegenerate symmetric bilinear form on a finitely generated free abelian group $L$. When, if ever, does there exist an infinite family of isomorphic finitely generated subgroups $\left(\Gamma_{\sigma}\right)_{\sigma \in \Sigma}$ of $\operatorname{Aut}_{\mathbb{Z}}(L,\langle\rangle$,$) such that for \sigma \neq \tau, \Gamma_{\sigma}$ is not conjugate to $\Gamma_{\tau}$ in $\operatorname{Aut}_{\mathbb{Z}}(L,\langle\rangle$,$) ?$

In this paper, we establish the existence of such infinite families $\left(\Gamma_{\sigma}\right)_{\sigma \in \Sigma}$ of nonconjugate isomorphic finitely generated subgroups when $(L,\langle\rangle$,$) splits as$ an orthogonal direct sum

$$
(L,\langle,\rangle) \cong\left(L_{1},\langle,\rangle\right) \perp\left(L_{2},\langle,\rangle\right) \perp\left(L_{3},\langle,\rangle\right)
$$

in which $\left(L_{1},\langle\rangle,\right)$ has signature $(2,1),\left(L_{2},\langle\rangle,\right)$ has signature $(n, 1)$ with $n \geq 2$, and $\left(L_{3},\langle\rangle,\right)$ is either zero or indefinite with $\operatorname{rk}_{\mathbf{z}}\left(L_{3}\right) \geq 3$. The parameter set $\Sigma$ may be thought of as an infinite subset of $\operatorname{Aut}_{\mathbf{z}}(L,\langle\rangle$,$) .$

The construction of the groups $\left(\Gamma_{\sigma}\right)_{\sigma \in \Sigma}$ uses a variation on the methods of our earlier paper [3]; in addition, the main theorem of [3] is needed to show finite generation. In $\S 1$, we recall some basic facts about orthogonal groups and integral quadratic forms. The necessary results from [3] are reviewed in $\S \S 2-3$, and the families $\left(\Gamma_{\sigma}\right)_{\sigma \in \Sigma}$ are constructed in $\S 4$ (Theorems 4.4 and 4.5).

Received by the editors August 26, 1991 and, in revised form, March 16, 1992.

1991 Mathematics Subject Classification. Primary 20E07, 20 E36. 
This work was done while the author was visiting the Max-Planck-Institut für Mathematik, Bonn. We wish to thank Christian Okonek for raising the question here considered. Especially we wish to thank Professor Hirzebruch and the staff of the MPI for their hospitality and help. In addition, we are grateful to the referee for pointing out some errors in an earlier draft, and for suggesting the use of a result of Millson [6] to simplify the discussion.

\section{INTEGRAL QUADRATIC FORMS AND THEIR ARITHMETIC SUBGROUPS}

Let $\langle\rangle:, L \times L \rightarrow \mathbb{Z}$ be a nondegenerate symmetric integral bilinear form on a free abelian group $L$ of finite rank $m$, say. $(L,\langle\rangle$,$) is said to be isotropic$ (over $\mathbb{Z}$ ) when there exists a nonzero element $\mathbf{x} \in L$ such that $\langle\mathbf{x}, \mathbf{x}\rangle=0$; otherwise $(L,\langle\rangle$,$) is said to be anisotropic. Put \Gamma=\operatorname{Aut}_{\mathbb{Z}}(L,\langle\rangle$,$) . The$ associated real form $\langle\rangle:, L \otimes \mathbb{R} \times L \otimes \mathbb{R} \rightarrow \mathbb{R}$ is diagonalisable as

$$
\sum_{i=1}^{p} x_{i} y_{i}-\sum_{i=p+1}^{p+q} x_{i} y_{i},
$$

assigning to $(L,\langle\rangle$,$) the signature (p, q)$ where $p+q=m ; \Gamma$ imbeds as a discrete subgroup of finite covolume in the group $\operatorname{Aut}_{\mathbb{R}}(L \otimes \mathbb{R},\langle\rangle,) \cong O(p, q)$, and acts properly discontinuously as a group of isometries of the symmetric space of $O(p, q)$. Moreover, $\Gamma$ is cocompact precisely when $\langle$,$\rangle is$ anisotropic. (When $L,\langle$,$\rangle ) is indefinite, a classical theorem of Meyer [5]$ asserts that for $\langle$,$\rangle to be anisotropic it is necessary that m \leq 4$.)

When the signature of $(L,\langle\rangle$,$) is (2,1)$, the corresponding symmetric space is the upper half-plane, so that $\Gamma$ is a Fuchsian group. When $(L,\langle\rangle$, is isotropic, $\Gamma$ contains a nonabelian free subgroup of finite index. When $(L,\langle\rangle$,$) is anisotropic, \Gamma$ contains, as a subgroup of finite index, a surface group $\Sigma_{g}^{+}$; that is, the fundamental group of an orientable surface of genus $g \geq 2$, having a presentation of the form

$$
\Sigma_{g}^{+}=\left\langle X_{1}, \ldots, X_{g}, Y_{1}, \ldots, Y_{g}: \prod_{r=1}^{g} X_{r} Y_{r} X_{r}^{-1} Y_{r}^{-1}\right\rangle .
$$

We summarise these observations.

Proposition 1.1. Let $\Gamma$ be the automorphism group of a nondegenerate integral quadratic form of signature $(2,1)$; then $\Gamma$ is finitely generated, and

(i) $\Gamma$ contains a surface subgroup of finite index when $(L,\langle\rangle$,$) is aniso-$ tropic;

(ii) $\Gamma$ contains a nonabelian free subgroup of finite index when $(L,\langle\rangle$, is isotropic.

Let $\mathbb{G}$ be a linear algebraic group defined and semisimple over $\mathbb{Q}$; we may take $\mathbb{G}$ to be imbedded $\mathbb{G}_{\mathbb{Q}} \subset \mathbb{G L}_{n}(\mathbb{Q})$. By an arithmetic subgroup of $\mathbb{G}$, we mean a subgroup $\Gamma$ of $\mathbb{G}_{\mathbb{R}}$ which is commensurable with $\mathbb{G}_{\mathbb{Z}}=\mathbb{G}_{\mathbb{Q}} \cap \mathbb{G L}_{n}(\mathbb{Z})$. This does not depend on the particular imbedding $\mathbb{G}_{\mathbb{Q}} \subset \mathbb{G L}_{n}(\mathbb{Q})$ chosen. Moreover, for such a subgroup $\Gamma, \mathbb{G}_{\mathbb{R}} / \Gamma$ has finite invariant volume. Let $\bar{\Delta} \subset \mathbb{G}_{\mathbb{C}}$ denote the Zariski closure of a subgroup $\Delta \subset \mathbb{G}_{\mathbb{R}}$.

We begin by observing the following, where $[\Gamma, \Gamma]$ denotes the commutator subgroup of $\Gamma$. 
Proposition 1.2. Let $\mathbb{G}$ be a linear algebraic group defined and semisimple over $\mathbb{Q}$, with the property that $\mathbb{G}_{i, \mathbb{R}}$ is noncompact for each $\mathbb{Q}$-simple factor $\mathbb{G}_{i}$. If $\Gamma$ is an arithmetic subgroup of $\mathbb{G}$ then $[\overline{\Gamma, \Gamma}]=\mathbb{G}_{\mathbb{C}}$.

Proof. We first consider the case where $\mathbb{G}$ is $\mathbb{Q}$-simple. By Borel's Density Theorem in the form of [1], $\bar{\Gamma}=\mathbb{G}_{\mathbb{C}}$, and since $\mathbb{G}_{\mathbb{C}}$ is nonabelian, $\Gamma$ is also nonabelian; hence $[\Gamma, \Gamma]$ is nontrivial. $\Gamma$ normalises $[\Gamma, \Gamma]$, so that $\bar{\Gamma}$ normalises $[\overline{\Gamma, \Gamma}]$. However, since $\bar{\Gamma}=\mathbb{G}_{\mathbb{C}},[\overline{\Gamma, \bar{\Gamma}}]$ is a normal complex algebraic subgroup of $\mathbb{G}_{\mathbb{C}}$. Moreover, since $[\overline{\Gamma, \Gamma}]$ is the Zariski closure of a subset $[\Gamma, \Gamma]$ of $\mathbb{G}_{\mathbb{Q}}$, then by Weil's Rationality Criterion $[8],[\overline{\Gamma, \Gamma}]$ is defined over $\mathbb{Q}$. The assertion that $[\overline{\Gamma, \Gamma}]=\mathbb{G}_{\mathbb{C}}$ now follows from the fact that $\mathbb{G}$ is $\mathbb{Q}$ simple and $[\overline{\Gamma, \Gamma}]$ is nontrivial.

In general, $\mathbb{G}$ is isogenous with the product of its $\mathbb{Q}$-simple factors $\mathbb{G}_{1} \times \cdots \times$ $\mathbb{G}_{n}$, so that $\Gamma$ contains, with finite index, a subgroup of the form $\Gamma_{1} \times \cdots \times \Gamma_{n}$, where $\Gamma_{i}$ is an arithmetic subgroup of $\mathbb{G}_{i}$. Hence $\left[\Gamma_{1}, \Gamma_{1}\right] \times \cdots \times\left[\Gamma_{n}, \Gamma_{n}\right]$ is contained in $[\Gamma, \Gamma]$, and the result follows easily from the special case already considered.

For any field $k$, let $O(n, k)$ denote the group of automorphisms of the standard symmetric bilinear form

$$
\langle,\rangle: k^{n} \times k^{n} \rightarrow k ; \quad\langle\mathbf{x}, \mathbf{y}\rangle=\sum_{i=1}^{n} x_{i} y_{i},
$$

and let $\mathfrak{O}(n, k)$ denote the Lie algebra of $O(n, k)$,

$$
\mathfrak{O}(n, k)=\left\{A \in M_{n}(k): A^{T}+A=0\right\} .
$$

The obvious isomorphism $k^{n_{1}} \oplus \cdots \oplus k^{n_{f}} \cong k^{n_{1}+\cdots+n_{f}}$ induces injections

$$
\mathfrak{O}\left(n_{1}, k\right) \oplus \cdots \oplus \mathfrak{O}\left(n_{f}, k\right) \subset \mathfrak{O}\left(n_{1}+\cdots+n_{f}, k\right),
$$

and

$$
O\left(n_{1}, k\right) \times \cdots \times O\left(n_{f}, k\right) \subset O\left(n_{1}+\cdots+n_{f}, k\right) .
$$

Proposition 1.3. $\mathfrak{O}\left(n_{1}, \mathbb{C}\right) \oplus \cdots \oplus \mathfrak{O}\left(n_{f}, \mathbb{C}\right)$ is a self-normalising Lie subalgebra of $\mathfrak{O}\left(n_{1}+\cdots+n_{f}, \mathbf{C}\right)$ provided that each $n_{i} \geq 2$.

Proof. It clearly suffices to show that $\mathfrak{O}(m, \mathbb{C}) \oplus \mathfrak{O}(n, \mathbb{C})$ is a self-normalizing Lie subalgebra of $\mathfrak{O}(m+n, \mathbb{C})$ provided that $m, n \geq 2$; the general case follows easily by induction. Thus suppose that $\alpha \in M_{m+n}(\mathbb{C})$ has the property

$(*) \quad[\alpha, \xi] \in \mathfrak{O}(m, \mathbb{C}) \oplus \mathfrak{O}(n, \mathbb{C}) \quad$ for all $\xi \in \mathfrak{O}(m, \mathbb{C}) \oplus \mathfrak{O}(n, \mathbb{C})$.

We may write $\alpha, \xi$ in block form:

$$
\alpha=\left[\begin{array}{ll}
A & B \\
C & D
\end{array}\right], \quad \xi=\left[\begin{array}{cc}
X & 0 \\
0 & Y
\end{array}\right]
$$

where $X \in \mathfrak{D}(m, \mathbb{C})$ and $Y \in \mathfrak{D}(n, \mathbb{C})$ so that

$$
[\alpha, \xi]=\left[\begin{array}{cc}
{[A, X]} & B Y-X B \\
C X-Y C & {[D, Y]}
\end{array}\right] .
$$

The condition that $[\alpha, \xi] \in \mathfrak{O}(m, \mathbb{C}) \oplus \mathfrak{O}(n, \mathbb{C})$ implies that $B Y-X B=0$ and $C X-Y C=0$. However, if $B Y-X B=0$ for all $X \in \mathfrak{O}(m, \mathbb{C})$ and all $Y \in \mathfrak{O}(n, \mathbb{C})$, then we may take $Y$ to be the zero matrix and, since $m \geq 2$, 
$X$ to be an invertible skew-symmetric matrix, from which we see immediately that $B=0$. Similarly $C=0$. If we now impose the additional condition that $\alpha \in \mathfrak{O}(m+n, \mathbb{C})$, that is, $\alpha^{T}+\alpha=0$, we see that $A^{T}+A=0$ and $D^{T}+D=0$. Hence $\alpha \in \mathfrak{O}(m, \mathbb{C}) \oplus \mathfrak{O}(n, \mathbb{C})$ as claimed.

For any group $G$ and subgroup $H$, we denote by $N_{G}(H)$ the normaliser of $H$ in $G$. When $\mathbb{G}$ is an algebraic group and $\mathbb{H}$ is an algebraic subgroup, $N_{\mathbb{G}}(\mathbb{H})$ is also an algebraic subgroup of $\mathbb{G}$. In particular, the normaliser $N\left(n_{1}, \ldots, n_{f}\right)$ of $O\left(n_{1}, \mathbb{C}\right) \times \cdots \times O\left(n_{f}, \mathbb{C}\right)$ in $O\left(n_{1}+\cdots+n_{f}, \mathbb{C}\right)$, is an algebraic subgroup of $O\left(n_{1}+\cdots+n_{f}, \mathbb{C}\right)$. It follows that $N\left(n_{1}, \ldots, n_{f}\right)$ is a complex Lie group; moreover, when each $n_{i} \geq 2$, it follows from Proposition 1.3 that $N\left(n_{1}, \ldots, n_{f}\right)$ has the same identity component as $O\left(n_{1}, \mathbb{C}\right) \times \cdots \times O\left(n_{f}, \mathbb{C}\right)$. Since any linear algebraic group over $\mathbb{C}$ has only finitely many connected components $[2$, p. 86]), we see that

Corollary 1.4. Let $N\left(n_{1}, \ldots, n_{f}\right)$ be the normaliser of $O\left(n_{1}, \mathbb{C}\right) \times \cdots \times O\left(n_{f}, \mathbb{C}\right)$ in $O\left(n_{1}+\cdots+f_{n}, \mathbb{C}\right)$; then $O\left(n_{1}, \mathbb{C}\right) \times \cdots \times O\left(n_{f}, \mathbb{C}\right)$ has finite index in $N\left(n_{1}, \ldots, n_{f}\right)$ provided that each $n_{i} \geq 2$.

Proposition 1.5. Let $L$ be a finitely generated free abelian group, and let $\langle$,$\rangle :$ $L \times L \rightarrow \mathbb{Z}$ be a nondegenerate symmetric integral bilinear form which splits as a direct sum

$$
(L,\langle,\rangle) \cong\left(L_{1},\langle,\rangle\right) \perp\left(L_{2},\langle,\rangle\right) \perp \cdots \perp\left(L_{f},\langle,\rangle\right)
$$

where $f \geq 2$, and each $\operatorname{rk}_{\mathbb{Z}}\left(L_{i}\right) \geq 2$. Let $\mathbb{G}$ (resp. $\mathbb{G}_{i}$ ) be the linear algebraic group whose group of $k$-rational points is $\operatorname{Aut}_{k}(L \otimes k,\langle\rangle$,$) , (resp.$ $\left.\operatorname{Aut}_{k}\left(L_{i} \otimes k,\langle\rangle,\right)\right)$, and let

$$
\mathbb{H}=\mathbb{G}_{1} \times \cdots \times \mathbb{G}_{f} \subset \mathbb{G} ;
$$

then $N_{\mathbb{G}}(\mathbb{H}) \cap \operatorname{Aut}_{\mathbb{Z}}(L,\langle\rangle$,$) contains \operatorname{Aut}_{\mathbb{Z}}\left(L_{1},\langle\rangle,\right) \times \cdots \times \operatorname{Aut}_{\mathbb{Z}}\left(L_{f},\langle\rangle,\right)$ as a subgroup of finite index.

Proof. Put $\lambda_{i}=\mathrm{rk}_{\mathbb{Z}}\left(L_{i}\right)$, and $\lambda=\sum \lambda_{i} . \mathbb{H}$ and $N_{\mathbb{G}}(\mathbb{H})$ are both linear algebraic subgroups of $\mathbb{G}$, defined over $\mathbb{Q}$, and the groups of real points, $\mathbb{H}_{\mathbb{R}}$ and $\left(N_{\mathbb{G}}(\mathbb{H})\right)_{\mathbf{R}}$ respectively, are Lie groups possessing only finitely many connected components. Observe that $\mathbb{G}_{\mathbb{C}}$ (respectively $\mathbb{G}_{i, \mathbb{C}}$ ) is isomorphic to $O(\lambda, \mathbb{C}$ ) (respectively $O\left(\lambda_{i}, \mathbb{C}\right)$ ), so that, by Corollary $1.4, \mathbb{H}_{\mathbb{C}}$ is a subgroup of finite index in $\left(N_{\mathbb{G}}(\mathbb{H})\right)_{\mathrm{C}}$. Thus the identity components of the corresponding real groups are equal; that is, $\mathbb{H}_{\mathbf{R}, 0}=\left(N_{\mathbb{G}}(\mathbb{H})\right)_{\mathbf{R}, 0}$. The conclusion follows since $\operatorname{Aut}_{\mathbf{Z}}\left(L_{1},\langle\rangle,\right) \times \cdots \times \operatorname{Aut}_{\mathbb{Z}}\left(L_{f},\langle\rangle,\right)$ and $N_{\mathbb{G}}(\mathbb{H}) \cap \operatorname{Aut}_{\mathbb{Z}}(L,\langle\rangle$,$) are both$ arithmetic in $N_{\mathbb{G}}(\mathbb{H})$, and $N_{\mathbb{G}}(\mathbb{H}) \cap \operatorname{Aut}_{\mathbb{Z}}(L,\langle\rangle$,$) contains \operatorname{Aut}_{\mathbb{Z}}\left(L_{1},\langle\rangle,\right) \times$ $\cdots \times \operatorname{Aut}_{\mathbb{Z}}\left(L_{f},\langle\rangle,\right)$.

\section{NORMAL SUBDIRECT PRODUCTS}

By a product structure on a group $G$ we mean a finite sequence $\mathscr{G}=\left(G_{r}\right)_{1 \leq r \leq n}$ of (nontrivial) normal subgroups of $G$ such that $G$ is the internal direct product $G=G_{1} \circ \ldots \circ G_{n}$; that is, each $g \in G$ can be expressed uniquely as a product $g=g_{1} \cdots g_{n}$ with $g_{i} \in G_{i}$. For a group $G$ having a product structure $\mathscr{G}=\left(G_{r}\right)_{1 \leq r \leq n}$, we identify $G$ with the external direct product $\prod_{j=1}^{n} G_{j}$. Let $\pi_{i}: \prod_{j=1}^{n} G_{j} \rightarrow G_{i}$ be the projection onto the $i$ th factor; a subgroup $H$ 
of $\prod_{i=1}^{n} G_{i}$ is a subdirect product of $G$ (or more accurately, of $\mathscr{G}$ ) when $\pi_{i}(H)=G_{i}$ for each $i$. Let $S\left(G_{1}, \ldots, G_{n}\right)$ the set of normal subdirect products of $G_{1} \circ \cdots \circ G_{n}$; that is, subdirect products which are also normal subgroups.

For any group $H$, let $\nu: H \rightarrow H^{\text {ab }}$ denote the canonical map onto the abelianisation $H^{\mathrm{ab}}=H /[H, H]$. To any product structure $\mathscr{G}=\left(G_{r}\right)_{1 \leq r \leq n}$, we may associate its abelianisation $\mathscr{G}^{\mathrm{ab}}=\left(G_{r}^{\mathrm{ab}}\right)_{1 \leq r \leq n}$. Moreover, the abelianisation map $\nu: G_{1} \circ \cdots \circ G_{n} \rightarrow G_{1}^{\mathrm{ab}} \circ \cdots \circ G_{n}^{\mathrm{ab}}$ induces a mapping

$$
\nu^{-1}: S\left(G_{1}^{\mathrm{ab}}, \ldots, G_{k}^{\mathrm{ab}}\right) \rightarrow S\left(G_{1}, \ldots, G_{k}\right)
$$

by means of $H \mapsto \nu^{-1}(H)$. We have shown elsewhere [3, Proposition 1.2] that

Proposition 2.1. For any product structure $\mathscr{G}=\left(G_{r}\right)_{1 \leq r \leq n}$

$$
\nu^{-1}: S\left(G_{1}^{\mathrm{ab}}, \ldots, G_{n}^{\mathrm{ab}}\right) \rightarrow S\left(G_{1}, \ldots, G_{n}\right)
$$

is bijective.

The following result of [3, Corollary 3.6] is important in the sequel.

Theorem 2.2. Let $H$ be a normal subdirect product of $G_{1} \circ \ldots \circ G_{n}$. Then $H$ is finitely generated (as a group, not merely as a normal subgroup) if and only if each $G_{i}$ is finitely generated.

The conclusion of Theorem 2.2 is false if the assumption of normality on $H$ is dropped.

\section{A CONSTRUCTION FOR ABELIAN SUBDIRECT PRODUCTS}

Let $B$ be an infinite finitely generated abelian group. By an oriented splitting for $B$, we shall mean a triple $X$ of the form $X=\left(M_{X}, N_{X}, \varepsilon_{X}\right)$, where $B / \operatorname{Tor}(B)=M_{X} \oplus N_{X}$ in which $N_{X}$ is free of rank 1 , and $\varepsilon_{X} \in N_{X}$ is a generator. We denote by $\mathfrak{S}(B)$ the set of oriented splittings of $B$. Clearly the group $\operatorname{Aut}(B / \operatorname{Tor}(B))$ acts transitively on $\mathfrak{S}(B)$. Since $\operatorname{Tor}(B)$ is a characteristic subgroup of $B$, there is a natural epimorphism $\operatorname{Aut}(B) \rightarrow \operatorname{Aut}(B / \operatorname{Tor}(B))$, from which we see that $\operatorname{Aut}(B)$ also acts transitively on $\mathfrak{S}(B)$.

We now consider subdirect products of abelian groups; it is more convenient to write our groups additively, and to confuse direct products with direct sums. Thus suppose that $A=A_{1} \oplus A_{2}$ where $A_{1}$ is a free abelian group of rank $r_{1} \geq 2$, and $A_{2}$ is a finitely generated abelian group such that $A_{2} / \operatorname{Tor}\left(A_{2}\right)$ has rank $r_{2} \geq 1$.

Let $X=\left(M_{X}, N_{X}, \varepsilon_{X}\right)$ be an oriented splitting for $A_{1}$, and $Y=\left(M_{Y}, N_{Y}\right.$, $\left.\varepsilon_{Y}\right)$ an oriented splitting for $A_{2} / \operatorname{Tor}\left(A_{2}\right)$. Let $\delta(X, Y)$ denote the subgroup of $A_{1} \oplus A_{2} / \operatorname{Tor}\left(A_{2}\right)$ defined by

$$
\delta(X, Y)=M_{X} \oplus\left\langle\varepsilon_{X}+\varepsilon_{Y}\right\rangle \oplus M_{Y},
$$

and let $\Delta(X, Y)$ denote the preimage of $\delta(X, Y)$ in $A=A_{1} \oplus A_{2}$, under the natural mapping

$$
\psi: A_{1} \oplus A_{2} \rightarrow A_{1} \oplus A_{2} / \operatorname{Tor}\left(A_{2}\right) .
$$

It is easy to see that $\Delta(X, Y)$ is a (necessarily normal) subdirect product of $A_{1} \oplus$ $A_{2}$. The group $\operatorname{Aut}\left(A_{1}, A_{2}\right)$ of product preserving automorphisms of $A_{1} \oplus A_{2}$ acts naturally on $S\left(A_{1}, A_{2}\right)$. Since $\operatorname{Aut}\left(A_{1}\right)$ imbeds naturally in $\operatorname{Aut}\left(A_{1}, A_{2}\right)$, by extending its natural action on $A_{1}$ via the identity on $A_{2}$, we see that 
$\operatorname{Aut}\left(A_{1}\right)$ also acts naturally on $S\left(A_{1}, A_{2}\right)$. On taking $\Delta=\Delta(X, Y)$ for some suitable oriented splittings $X=\left(M_{X}, N_{X}, \varepsilon_{X}\right)$ and $Y=\left(M_{Y}, N_{Y}, \varepsilon_{Y}\right)$ for $A_{1}$ and $A_{2} / \operatorname{Tor}\left(A_{2}\right)$ respectively, we obtain

Theorem 3.1. Let $A_{1}, A_{2}$ be finitely generated abelian groups such that $A_{1}$ is free abelian of rank $r_{1} \geq 2$, and $A_{2} / \operatorname{Tor}\left(A_{2}\right)$ has rank $r_{2} \geq 1$. Then there is a subdirect product $\Delta \subset A_{1} \oplus A_{2}$, and an infinite subset $\Theta \subset \operatorname{Aut}\left(A_{1}\right)$ such that $\theta(\Delta) \neq \sigma(\Delta)$ for $\theta, \sigma \in \Theta$ such that $\theta \neq \sigma$.

\section{INFINITE FAMILIES OF NONCONJUGATE ISOMORPHIC IMBEDDINGS}

Let $\Lambda_{1}$ be a nonabelian free group of finite rank $m \geq 2$, and let $\Lambda_{2}$ be a finitely generated group such that $\Lambda_{2}^{\mathrm{ab}}$ is infinite. Put $A_{i}=\Lambda_{i}^{\mathrm{ab}}$ for $i=1,2$. Since $A_{1} \cong \mathbb{Z}^{m}$ and $A_{2}$ maps epimorphically onto $\mathbb{Z}$, we may apply Theorem 3.1 to obtain the existence of a faithfully indexed family $(\theta(\Delta))_{\theta \in \Theta}$ of normal subdirect products of $A_{1} \oplus A_{2}$, where $\theta$ ranges over some infinite subset $\theta$ of $\operatorname{Aut}\left(A_{1}\right) \cong \mathrm{GL}_{m}(\mathbb{Z})$. As we have seen, $\nu^{-1}: S\left(A_{1}^{\mathrm{ab}}, A_{2}^{\mathrm{ab}}\right) \rightarrow S\left(\Lambda_{1}, \Lambda_{2}\right)$ is bijective. Put $\Gamma=\nu^{-1}(\Delta)$; then $\Gamma$ is a normal subdirect product of $\Lambda_{1} \times \Lambda_{2}$, and so is finitely generated by Theorem 2.2. Furthermore, the group $\operatorname{Aut}\left(\Lambda_{1}\right) \times$ $\operatorname{Aut}\left(\Lambda_{2}\right)$ acts naturally on subgroups of $\Lambda_{1} \times \Lambda_{2}$, and the orbit of $\Gamma$ under this action consists entirely of normal subdirect products of $\Lambda_{1} \times \Lambda_{2}$. In fact, we need only consider the subgroup $\operatorname{Aut}\left(\Lambda_{1}\right) \cong \operatorname{Aut}\left(\Lambda_{1}\right) \times\{1\}$ of $\operatorname{Aut}\left(\Lambda_{1}\right) \times$ Aut $\left(\Lambda_{2}\right)$. Since $\Lambda_{1}$ is free, by a theorem of Nielsen [7], for each automorphism $\theta$ of $A_{1}=\Lambda_{1}^{\mathrm{ab}}$ we may choose a lifting of $\theta$ to an automorphism $\hat{\theta}$ of $\Lambda_{1} \cong$ $\Lambda_{1} \times\{1\}$. Put $\Gamma_{\theta}=\hat{\theta}(\Gamma)$. It is clear that $\Gamma_{\theta}$ is isomorphic to $\Gamma$. We may summarise our progress so far thus:

Theorem 4.1. Let $\Lambda_{1}$ be a nonabelian free group of finite rank $m \geq 2$, and let $\Lambda_{2}$ be a finitely generated group which maps epimorphically onto $\mathbb{Z}$; then there is a subset $\boldsymbol{\theta} \subset \operatorname{Aut}\left(A_{1}\right)$ which parametrises an infinite family $\left(\Gamma_{\theta}\right)_{\theta \in \Theta}$ of mutually isomorphic finitely generated normal subdirect products of $\Lambda_{1} \times \Lambda_{2}$ with the property that $\Gamma_{\theta} \neq \Gamma_{\sigma}$ for $\theta \neq \sigma$.

The analogue of Theorem 4.1 in which $\Lambda_{1}$ is replaced by the fundamental group of a closed orientable surface is also true; we proceed to outline the necessary variations.

Let $\Sigma_{+}^{g}$ denote the closed orientable surface of genus $g \geq 2$, and let $\Sigma_{g}^{+}$ denote its fundamental group;

$$
\Sigma_{g}^{+}=\left\langle X_{1}, \ldots, X_{g}, Y_{1}, \ldots, Y_{g}: \prod_{r=1}^{g} X_{r} Y_{r} X_{r}^{-1} Y_{r}^{-1}\right\rangle .
$$

We may identify the abelianisation $H_{1}\left(\Sigma_{g}^{+} ; \mathbb{Z}\right)$ of $\Sigma_{g}^{+}$with $\mathbb{Z}^{2 g}$, and the intersection form on $\Sigma_{+}^{g}$ gives rise to a nondegenerate skew-symmetric bilinear form $\langle\rangle:, \mathbb{Z}^{2 g} \times \mathbb{Z}^{2 g} \rightarrow \mathbb{Z}$. With this identification, symplectic automorphisms of $\mathbb{Z}^{2 g}$ lift back to automorphisms of $\Sigma_{g}^{+}=\pi_{1}\left(\Sigma_{+}^{g}\right)$, with transvections lifting back to Dehn twists.

Let $\left\{\varepsilon_{1}, \ldots, \varepsilon_{g}, \phi_{1}, \ldots, \phi_{g}\right\}$ be the standard symplectic basis for $\langle$,$\rangle ;$ that is,

$$
\left\langle\varepsilon_{i}, \varepsilon_{j}\right\rangle=\left\langle\phi_{i}, \phi_{j}\right\rangle=0 ; \quad\left\langle\varepsilon_{i}, \phi_{j}\right\rangle=\delta_{i j} .
$$


In constructing subdirect products in $A_{1} \oplus A_{2}$, as in $\S 3$, where now $A_{1}=$ $H_{1}\left(\Sigma_{g}^{+} ; \mathbb{Z}\right) \cong \mathbb{Z}^{2 g}$, we take our "basepoint splitting" $X$ of $A_{1}$ to be of the form $A_{1}=M_{X} \oplus N_{X}$, where $\operatorname{Span}_{\mathbb{Z}}\left\{\varepsilon_{1}, \ldots, \varepsilon_{g}\right\} \subset M_{X}$ and $N_{X} \subset\left\{\phi_{1}, \ldots, \phi_{g}\right\}$. There is an infinite set of such splittings which we parametrise by suitable elements of the group $\mathrm{Sp}_{2 g}(\mathbb{Z})$. With these modifications, we obtain the following analogue of Theorem 4.1 .

Theorem 4.2. Let $\Lambda_{1}$ be a surface group of genus $g \geq 2$, and let $\Lambda_{2}$ be a finitely generated group which maps epimorphically onto $\mathbb{Z}$; then there is a subset $\boldsymbol{\Theta} \subset \mathrm{Sp}_{2 g}(\mathbb{Z})$ which parametrises an infinite family $\left(\Gamma_{\theta}\right)_{\theta \in \Theta}$ of mutually isomorphic finitely generated normal subdirect products of $\Lambda_{1} \times \Lambda_{2}$ with the property that $\Gamma_{\theta} \neq \Gamma_{\sigma}$ for $\theta \neq \sigma$.

Theorem 4.3. Let $\langle\rangle:, L \times L \rightarrow \mathbb{Z}$ be a nondegenerate symmetric bilinear form on a finitely generated free abelian group $L$, such that $(L,\langle\rangle$,$) splits as an$ orthogonal direct sum

$$
(L,\langle,\rangle) \cong\left(L_{1},\langle,\rangle\right) \perp\left(L_{2},\langle,\rangle\right),
$$

where $\left(L_{1},\langle\rangle,\right)$ has signature $(2,1)$, and $\operatorname{Aut}_{\mathbb{Z}}\left(L_{2},\langle\rangle,\right)$ has a subgroup of finite index which maps epimorphically onto $\mathbb{Z}$. Then there exists an infinite family $\left(\Gamma_{\sigma}\right)_{\sigma \in \Sigma}$ of mutually isomorphic finitely generated nonconjugate subgroups of $\operatorname{Aut}_{\mathbb{Z}}\left(L_{1},\langle\rangle,\right) \times \operatorname{Aut}_{\mathbb{Z}}\left(L_{2},\langle\rangle,\right)$.

Proof. $\operatorname{Aut}_{\mathbb{Z}}\left(L_{i},\langle\rangle,\right)$ is a finitely generated linear group, and so, by Selberg's Theorem, has a torsion free subgroup, $\Lambda_{i}$ say, of finite index. Suppose that $\left(L_{1},\langle\rangle,\right)$ has signature $(2,1)$; if $\left(L_{1},\langle\rangle,\right)$ is isotropic, then $\Lambda_{1}$ is free, whilst if $\left(L_{1},\langle\rangle,\right)$ is anisotropic, then $\Lambda_{1}$ is a surface group. Either way, if $\Lambda_{2}$ maps epimorphically onto $\mathbb{Z}$, we may apply the results of Theorems 4.1 and 4.2 to conclude that there is an infinite family, $\left(\Gamma_{\delta}\right)_{\theta \in \Theta}$, of mutually isomorphic finitely generated normal subdirect products of $\Lambda_{1} \times \Lambda_{2}$. Moreover, since the family $\left(\Gamma_{\theta}\right)_{\theta \in \Theta}$ consists of normal subgroups of $\Lambda_{1} \times \Lambda_{2}$, we see that no $\Gamma_{\theta}$ is conjugate in $\Lambda_{1} \times \Lambda_{2}$ to any $\Gamma_{\sigma}$ for $\theta \neq \sigma$.

Since $\Lambda_{1} \times \Lambda_{2}$ has finite index in $\operatorname{Aut}_{\mathbb{Z}}\left(L_{1},\langle\rangle,\right) \times \operatorname{Aut}_{\mathbb{Z}}\left(L_{2},\langle\rangle,\right)$, each $\Gamma_{\theta}$ is conjugate in $\operatorname{Aut}_{\mathbb{Z}}\left(L_{1},\langle\rangle,\right) \times \operatorname{Aut}_{\mathbb{Z}}\left(L_{2},\langle\rangle,\right)$ to at most finitely many $\Gamma_{\sigma}$. In particular, we may choose an infinite subfamily $\left(\Gamma_{\sigma}\right)_{\sigma \in \Sigma}$, so that no two distinct elements are conjugate in $\operatorname{Aut}_{\mathbb{Z}}\left(L_{1},\langle\rangle,\right) \times \operatorname{Aut}_{\mathbb{Z}}\left(L_{2},\langle\rangle,\right)$.

Although not conjugate in $\operatorname{Aut}_{\mathbb{Z}}\left(L_{1},\langle\rangle,\right) \times \operatorname{Aut}_{\mathbb{Z}}\left(L_{2},\langle\rangle,\right)$, subgroups in the family $\left(\Gamma_{\sigma}\right)_{\sigma \in \Sigma}$ just constructed may become conjugate in $\operatorname{Aut}_{\mathbb{Z}}(L,\langle\rangle$,$) .$ We show, however, that for each $\tau \in \Sigma$, the set $\left\{\sigma \in \Sigma: \Gamma_{\sigma}\right.$ is conjugate to $\Gamma_{\tau}$ in $\left.\operatorname{Aut}_{\mathbb{Z}}(L,\langle\rangle),\right\}$ is finite.

Theorem 4.4. Let $\langle\rangle:, L \times L \rightarrow \mathbb{Z}$ be a nondegenerate symmetric bilinear form on a finitely generated free abelian group $L$, such that $(L,\langle\rangle$,$) splits as an$ orthogonal direct sum

$$
(L,\langle,\rangle) \cong\left(L_{1},\langle,\rangle\right) \perp\left(L_{2},\langle,\rangle\right)
$$

where $\left(L_{1},\langle\rangle,\right)$ has signature $(2,1)$, and $\operatorname{Aut}_{\mathbb{Z}}\left(L_{2},\langle\rangle,\right)$ has a subgroup of finite index which maps epimorphically onto $\mathbb{Z}$. Then there exists an infinite family $\left(\Gamma_{\omega}\right)_{\omega \in \Omega}$ of mutually isomorphic finitely generated subgroups of $\operatorname{Aut}_{\mathbb{Z}}(L,\langle\rangle$,$) such that \Gamma_{\omega}$ is not conjugate, in $\operatorname{Aut}_{\mathbb{Z}}(L,\langle\rangle$,$) , to \Gamma_{\mu}$ for $\omega \neq \mu$. 
Proof. Let $\mathbb{G}$ (resp. $\mathbb{G}_{i}$ ) be the linear algebraic group whose group of $k$ rational points is $\operatorname{Aut}_{k}(L \otimes k,\langle\rangle$,$\left.) (resp. \operatorname{Aut}_{k}\left(L_{i} \otimes k,\langle\rangle,\right)\right)$, and let $\mathbb{H}=\mathbb{G}_{1} \times \mathbb{G}_{2} \subset \mathbb{G}$. Let $\Gamma_{\sigma}, \Gamma_{\tau}$ be subgroups from the family constructed in Theorem 4.3, and suppose that for some $g \in \operatorname{Aut}_{\mathbb{Z}}(L,\langle\rangle),, g \Gamma_{\sigma} g^{-1}=\Gamma_{\tau}$. Since $\Gamma_{\sigma}, \Gamma_{\tau}$ are normal subdirect products of $\Lambda_{1} \times \Lambda_{2}$, then by [3],

$$
\left[\Lambda_{1}, \Lambda_{1}\right] \times\left[\Lambda_{2}, \Lambda_{2}\right] \subset \Gamma_{\sigma} \cap \Gamma_{\tau} .
$$

Since $\left(L_{1},\langle\rangle,\right)$ has signature $(2,1)$, it follows that $\mathbb{G}_{1}$ is $\mathbb{Q}$-simple, and $\mathbb{G}_{1, \mathbb{R}}$ is noncompact. The condition that $\operatorname{Aut}_{\mathbb{Z}}\left(L_{2},\langle\rangle,\right)$ has a subgroup of finite index which maps epimorphically onto $\mathbb{Z}$ implies that $\left(L_{2},\langle\rangle,\right)$ is indefinite, and that $\operatorname{rk}_{\mathbb{Z}}\left(L_{2}\right) \geq 3$. If $\operatorname{rk}_{\mathbb{Z}}\left(L_{2}\right) \neq 4$ then $\mathbb{G}_{2}$ is $\mathbb{Q}$-simple, and $\mathbb{G}_{2, \mathbb{R}}$ is noncompact. If $\operatorname{rk}_{\mathbb{Z}}\left(L_{2}\right)=4$ then either $\mathbb{G}_{2}$ is $\mathbb{Q}$-simple, and $\mathbb{G}_{2, \mathbb{R}}$ is noncompact, or $\mathbb{G}_{2}$ is a product $\mathbb{L}_{1} \times \mathbb{L}_{2}$ where $\mathbb{L}_{1}$ and $\mathbb{L}_{2}$ are both $\mathbb{Q}$ simple, and $\mathbb{L}_{1, \mathbb{R}}, \mathbb{L}_{2, \mathbb{R}}$ are both noncompact. Either way, if $\mathbb{L}$ is a $\mathbb{Q}$-simple factor of $\mathbb{G}_{1} \times \mathbb{G}_{2}$, then $\mathbb{L}_{\mathbb{R}}$ is noncompact; applying (1.2) we conclude that $\left[\overline{\Lambda_{i}, \Lambda_{i}}\right]=\mathbb{G}_{i}$. Thus $\left[\overline{\Lambda_{1}, \Lambda_{1}}\right] \times\left[\overline{\Lambda_{2}, \Lambda_{2}}\right]=\mathbb{H}$. It now follows that $g \in$ $N_{\mathbb{G}}(\mathbb{H}) \cap \operatorname{Aut}_{\mathbb{Z}}(L,\langle\rangle$,$) .$

Denote the index of $\operatorname{Aut}_{\mathbb{Z}}\left(L_{1},\langle\rangle,\right) \times \operatorname{Aut}_{\mathbb{Z}}\left(L_{2},\langle\rangle,\right)$ in $N_{\mathbb{G}}(\mathbb{H}) \cap$ $\operatorname{Aut}_{\mathbb{Z}}(L,\langle\rangle$,$) by \alpha$. For each $\tau \in \Sigma$, the set $C_{\tau}=\left\{\sigma \in \Sigma: \Gamma_{\sigma}\right.$ is conjugate to $\Gamma_{\tau}$ in $\left.\operatorname{Aut}_{\mathbb{Z}}(L,\langle\rangle),\right\}$ has cardinality bounded by $\alpha$. By (1.5), $\alpha$ is finite, so that each $C_{\tau}$ is finite. Let $\Omega$ be a subset of $\Sigma$ obtained by choosing exactly one element from each $C_{\tau}$; then $\Omega$ is infinite, and the family $\left(\Gamma_{\omega}\right)_{\omega \in \Omega}$ consists of isomorphic finitely generated subgroups of $\operatorname{Aut}_{\mathbb{Z}}(L,\langle\rangle$,$) , and has$ the desired property that $\Gamma_{\omega}$ is not conjugate, in $\operatorname{Aut}_{\mathbb{Z}}(L,\langle\rangle$,$) , to \Gamma_{\mu}$ for $\omega \neq \mu$.

Analogously, we show

Theorem 4.5. Let $\langle\rangle:, L \times L \rightarrow \mathbb{Z}$ be a nondegenerate symmetric bilinear form on a finitely generated free abelian group $L$, such that $(L,\langle\rangle$,$) splits as an$ orthogonal direct sum

$$
(L,\langle,\rangle) \cong\left(L_{1},\langle,\rangle\right) \perp\left(L_{2},\langle,\rangle\right) \perp\left(L_{3},\langle,\rangle\right)
$$

where $\left(L_{1},\langle\rangle,\right)$ has signature $(2,1), \operatorname{Aut}_{\mathbb{Z}}\left(L_{2},\langle\rangle,\right)$ has a subgroup of finite index which maps epimorphically onto $\mathbb{Z}$, and where $\left(L_{3},\langle\rangle,\right)$ is indefinite with $\mathrm{rk}_{\mathbb{Z}}\left(L_{3}\right) \geq 3$. Then there exists an infinite family $\left(\Delta_{\omega}\right)_{\omega \in \Omega}$ of mutually isomorphic finitely generated nonconjugate subgroups of $\operatorname{Aut}_{\mathbb{Z}}(L,\langle\rangle$,$) .$

Proof. Let $\left(\Gamma_{\sigma}\right)_{\sigma \in \Sigma}$ be the family of mutually isomorphic finitely generated nonconjugate subgroups of $\operatorname{Aut}_{\mathbb{Z}}\left(L_{1},\langle\rangle,\right) \times \operatorname{Aut}_{\mathbb{Z}}\left(L_{2},\langle\rangle,\right)$ constructed in Theorem 4.3, and for each $\sigma \in \Sigma$, put

$\Delta_{\sigma}=\Gamma_{\sigma} \times \operatorname{Aut}_{\mathbb{Z}}\left(L_{3},\langle\rangle,\right) \subset \operatorname{Aut}_{\mathbb{Z}}\left(L_{1},\langle\rangle,\right) \times \operatorname{Aut}_{\mathbb{Z}}\left(L_{2},\langle\rangle,\right) \times \operatorname{Aut}_{\mathbb{Z}}\left(L_{3},\langle\rangle,\right)$.

Let $\mathbb{G}$ (resp. $\mathbb{G}_{i}$ ) be the linear algebraic group whose group of $k$-rational points is $\operatorname{Aut}_{k}(L \otimes k,\langle\rangle$,$) (resp. \operatorname{Aut}_{k}\left(L_{i} \otimes k,\langle\rangle,\right)$ ), and let $\mathbb{H}=\mathbb{G}_{1} \times$ $\mathbb{G}_{2} \times \mathbb{G}_{3} \subset \mathbb{G}$. As in the proof of Theorem 4.4, we obtain $\left[\overline{\Lambda_{1}, \Lambda_{1}}\right] \times\left[\overline{\Lambda_{2}, \Lambda_{2}}\right]=$ $\mathbb{G}_{1} \times \mathbb{G}_{2}$.

If $\operatorname{rk}_{\mathbb{Z}}\left(L_{3}\right) \neq 4$ then $\mathbb{G}_{3}$ is $\mathbb{Q}$-simple, and $\mathbb{G}_{3, \mathbb{R}}$ is noncompact. If $\operatorname{rk}_{\mathbb{Z}}\left(L_{3}\right)=$ 4 then, since $\left(L_{3},\langle\rangle,\right)$ is indefinite, either the identity component of $\mathbb{G}_{3, \mathbb{R}}$ is isomorphic to $\mathrm{SO}(3,1)$ and $\mathbb{G}_{3}$ is $\mathbb{Q}$-simple, or $\mathbb{G}_{3, \mathbb{R}}$ is locally isomorphic to a product $\mathrm{SO}(2,1) \times \mathrm{SO}(2,1)$; either way, if $\mathbb{L}$ is a $\mathbb{Q}$-simple factor of $\mathbb{G}$, 
then $\mathbb{L}_{\mathbb{R}}$ is noncompact, so that we may apply Proposition 1.2 to conclude that $\left[\overline{\Lambda_{3}, \Lambda_{3}}\right]=\mathbb{G}_{3}$, and

$$
\left[\overline{\Lambda_{1}, \Lambda_{1}}\right] \times\left[\overline{\Lambda_{2}, \Lambda_{2}}\right] \times\left[\overline{\Lambda_{3}, \Lambda_{3}}\right]=\mathbb{G}_{1} \times \mathbb{G}_{2} \times \mathbb{G}_{3} .
$$

As in the proof of Theorem 4.4, for each $\tau \in \Sigma$, the cardinality of the set

$$
C_{\tau}=\left\{\sigma \in \Sigma: \Delta_{\sigma} \text { is conjugate to } \Delta_{\tau} \text { in } \operatorname{Aut}_{\mathbb{Z}}(L,\langle,\rangle)\right\}
$$

is bounded by the index, $\alpha$, of $\operatorname{Aut}_{\mathbb{Z}}\left(L_{1},\langle\rangle,\right) \times \operatorname{Aut}_{\mathbb{Z}}\left(L_{2},\langle\rangle,\right) \times \operatorname{Aut}_{\mathbb{Z}}\left(L_{3},\langle\rangle,\right)$ in $N_{\mathbb{G}}(\mathbb{H}) \cap \operatorname{Aut}_{\mathbb{Z}}(L,\langle\rangle$,$) . By Proposition 1.5, \alpha$ is finite, so that each $C_{\tau}$ is finite. Let $\Omega$ be a subset of $\Sigma$ obtained by choosing exactly one element from each $C_{\tau}$; then $\Omega$ is infinite, and the family $\left(\Delta_{\omega}\right)_{\omega \in \Omega}$ consists of isomorphic finitely generated subgroups of $\operatorname{Aut}_{\mathbb{Z}}(L,\langle\rangle$,$) , and has the desired property$ that $\Delta_{\omega}$ is not conjugate, in $\operatorname{Aut}_{\mathbb{Z}}(L,\langle\rangle$,$) , to \Delta_{\mu}$ for $\omega \neq \mu$.

The referee points out that the condition "Aut ${ }_{\mathbb{Z}}\left(L_{2},\langle\rangle,\right)$ has a subgroup of finite index which maps epimorphically onto $\mathbb{Z}$ ", is precisely the same as requiring that $\left(L_{2},\langle\rangle,\right)$ have signature $(n, 1)$ for some $n \geq 2$. Indeed, if $\operatorname{Aut}_{\mathbb{Z}}\left(L_{2},\langle\rangle,\right)$ has a subgroup $\Gamma$ of finite index which maps epimorphically onto $\mathbb{Z}$, then $H_{1}(\Gamma, \mathbb{Z})$ is infinite, so that, by Kazhdan's Theorem [4], $\left(L_{2},\langle\rangle,\right)$ has signature $(n, 1)$ for some $n \geq 2$. Conversely, Millson [6, §4] has shown that for any nondegenerate integral quadratic form $(L,\langle\rangle$,$) of$ signature $(n, 1)$, with $n \geq 2$, there exists a subgroup $\Gamma$ of finite index in $\operatorname{Aut}_{\mathbb{Z}}(L,\langle\rangle$,$) for which H_{1}(\Gamma, \mathbb{Z})$ is infinite; in particular, $\Gamma$ maps epimorphically onto $\mathbb{Z}$. Combining this observation with Theorems 4.4 and 4.5 , we obtain

Corollary 4.6. Let $\langle\rangle:, L \times L \rightarrow \mathbb{Z}$ be a nondegenerate symmetric bilinear form on a finitely generated free abelian group $L$, such that $(L,\langle\rangle$,$) splits as$ an orthogonal direct sum

$$
(L,\langle,\rangle) \cong\left(L_{1},\langle,\rangle\right) \perp\left(L_{2},\langle,\rangle\right) \perp\left(L_{3},\langle,\rangle\right)
$$

where $\left(L_{1},\langle\rangle,\right)$ has signature $(2,1),\left(L_{2},\langle\rangle,\right)$ has signature $(n, 1)$ for some $n \geq 2$, and either $L_{3}=0$ or $\left(L_{3},\langle\rangle,\right)$ is indefinite with $\operatorname{rk}_{\mathbb{Z}}\left(L_{3}\right) \geq 3$. Then there exists an infinite family $\left(\Delta_{\omega}\right)_{\omega \in \Omega}$ of mutually isomorphic finitely generated nonconjugate subgroups of $\operatorname{Aut}_{\mathbb{Z}}(L,\langle\rangle$,$) .$

Our concern in this paper has been with conjugacy of subgroups within the discrete group $\operatorname{Aut}_{\mathbb{Z}}(L,\langle\rangle$,$) . From a different viewpoint, our results can$ be seen as a failure of the rigidity property for subgroups of infinite covolume within the corresponding Lie group $\operatorname{Aut}_{\mathbb{R}}\left(L \otimes_{\mathbb{Z}} \mathbb{R},\langle,\rangle \otimes 1\right)$; recall that the groups $\Gamma_{\sigma}$ we construct all have infinite index in $\operatorname{Aut}_{\mathbb{Z}}(L,\langle\rangle$,$) . If G$ is a noncompact linear almost simple Lie group with $\operatorname{rank}_{\mathbb{R}} \geq 2$, then in consequence of the super-rigidity theorem of Margulis, when $\Delta$ is a discrete subgroup of finite covolume in $G$ there are only finitely many $G$-conjugacy classes of discrete finitely generated subgroups isomorphic to $\Delta$. The arguments of the present paper can be extended to show that under the hypothesis " $\Delta$ is finitely generated, discrete, of infinite covolume in $G$ ", the number of $G$-conjugacy classes of discrete finitely generated subgroups isomorphic to $\Delta$ becomes infinite in general. We will pursue this idea more fully elsewhere. 


\section{REFERENCES}

1. A. Borel, Density properties of certain subgroups of semisimple groups, Ann. of Math. (2) 72 (1960), 179-188.

2. __ Linear algebraic groups, Benjamin, New York, 1969.

3. F. E. A. Johnson, On normal subgroups of direct products, Proc. Edinburgh Math. Soc. 33 (1990), 309-319.

4. D. A. Kahzdan, On the connection between the dual space of a group and the structure of its closed subgroups, Functional Anal. Appl. 1 (1967), 63-65.

5. A. Meyer, Zur Theorie der indefiniten quadratischen Formen, J. Reine Angew. Math. (1891), 125-139.

6. J. J. Millson, On the first Betti number of a constant negatively curved manifold, Ann. of Math. (2) (1976), 235-247.

7. J. Nielsen, Die Isomorphismengruppe der freien Gruppen, Math. Ann. 91 (1924), 169-209.

8. A. Weil, The field of definition of a variety, Amer. J. Math. 78 (1956), 509-524.

Department of Mathematics, University College London, London WCIE 6BT, United KINGDOM

E-mail address: ucahfea@ucl.ac.uk 